\section{Hierarchy of behaviours in a gastropod}

from Marian Dawkins

Animal Behaviour Correspondent

ANIMALS in their natural environments are often exposed to mixed, even contradictory sensory inputs. It may be impossible for an animal to react to all these inputs at once, as the behaviours involved may actually be mutually incompatible; for example, an animal cannot both approach and withdraw from an object at the same time. One of the interesting features of the evolution of the behaviour of animals has therefore been the development of a system of priorities whereby these conflicts are resolved in such a way that the survival and reproduction of the animal is most likely. This often takes the form of animals in conflict. situations performing certain behavioural responses to the partial or complete exclusion of others, and in this sense the animals can be said to have a hierarchy of behaviours.

Marine molluscs such as Pleurobranchaea have already proved to be extremely fruitful material for understanding the neurophysiological bases of many behaviours and it is therefore promising for the discovery of the neural basis of such a behavioural priority system that their behaviour has been found to be organised into an explicit priority sequence. Davis, Mpitsos and Pinneo (J. comp. Physiol., 90, 207-224; 225-243; 1974) have found that in this species, which is a carnivorous gastropod, feeding is a relatively dominant behaviour and takes precedence over most other behaviours. They presented animals with stimuli which released two different behaviours: in one experiment they gave a food stimulus to animals which had been turned upside down. Instead of showing their usual almost immediate righting behaviour, the animals stayed on their backs and ate, often for up to an hour. Feeding also dominated head withdrawal and mating, and Davis et al. argue that the adaptive significance of this is that Pleurobranchaea is a carnivore with a sporadic and rather limited food supply and so opportunities for feeding being rather scarce, feeding requires immediate and decisive interruption of all other behaviours.

Interestingly, there are some situations in which feeding is not given top priority. Davis et al. found that this behaviour is hormonally suppressed during egg laying; presumably this is an adaptation to ensure that the animals do not eat their own eggs. Even higher in the hierarchy than egg laying is the escape swimming response to avoid predators. This can interrupt any other behavioural act and so ensures that the animals survive to perform their other behaviours at times when no danger threatens.

The organisation of behaviours into hierarchies of adaptive priorities, Davis et al. suggest, may be a unifying theme for a diversity of complex behavioural phenomena in many animals, and to have discovered one in a mollusc with a simple analysable nervous system encourages the analysis of this phenomenon at the level of the single nerve cell.

\section{Can partons be saved?}

from David J. Miller

THE most eagerly anticipated parts of the International Conference on High Energy Physics, held in London during the first ten days of July, were the sessions devoted to electron-positron annihilation. Most delegates had already heard of the surprising results obtained by physicists at Stanford California, and at Cambridge Massachusets, concerning the rate of production of strongly interacting particles (hadrons). The conference gave the Stanford group a chance to report their results in detail, and to be closely crossquestioned about them. It also gave theorists their first chance to try and explain what had been discovered.

The Stanford and Cambridge experiments involved the storage of electron and positron beams of up to $2.6 \mathrm{GeV}$. The stored beams were brought together, twice per circuit in the Stanford SPEAR ring, and allowed to make head-on collisions. There was an array of scintillation counters and spark chambers around each intersection region, and it was possible to recognise whether outgoing charged tracks were caused by electrons, muons or hadrons. Electrons produce characteristic showers when they pass through dense material; hadrons are totally absorbed by a foot or so of steel, because of their strong interactions; muons are more penetrating than any other charged narticles. The American results confirmed findings, at Frascati in Italy, Orsay in France and at Novosibirsk in Russia, that electron-positron scattering has all the properties predicted for it by the well established theory of quantum-electrodynamics. Elastic scattering. the production of muon pairs and the production of photon pairs all behaved exactly according to theory. The surprise came in the rate of hadron production. Up to a beam energy of about $1.5 \mathrm{GeV}$ at Frascati, this rate falls off in the same way as the production of muon pairs. But in going from a total electron-positron energy of $3 \mathrm{GeV}$ (that is $1.5 \mathrm{GeV}$ electrons plus $1.5 \mathrm{GeV}$ positrons, meeting head on) to the $5.2 \mathrm{GeV}$ available at Stanford and Cambridge, the rate of hadron production levelled off and stayed roughly constant. This is not a contradiction of quantum electrodynamics, but it is very surprising.

Theorists had been quite happy with the behaviour of hadron production at the lower energies. They had compared it with muon production, and worked out a theory in which the electron and positron annihilated by first of all creating a massive single photon. This process is formally just the reverse of electron-positron pair production by a photon close to an atomic nucleus. The heavy photon could then generate a muon pair, one positive and one negative, or it might generate a 'parton' pair in just the same way. Partons are hypothetical objects which are supposed to be the building blocks from which the hadrons are made-just as the hadrons themselves are the building blocks for nuclei.

The parton theories have been supported by a number of recent experiments where leptons (electrons, muons or neutrinos) are scattered from nucleons (protons or neutrons, free or in nuclei). These so called deep inelastic scattering experiments have been done with electrons at a number of laboratories, including Stanford, and with neutrinos in the Gargamelle bubble chamber at CERN, Geneva. The results are in some ways similar to Geiger and Marsden's alpha particle scattering experiment, which led Rutherford in 1911 to postulate a small point nucleus in each atom. The angular distribution in deep inelastic lepton scattering from a proton is just what would be expected if protons had very small constituents. There is even evidence to suggest that these constituents or partons may have the same charge and spin as the quarks, which were postulated in 1973 by Gell-Mann and Zweig to explain the regularities in the spectrum of the resonances of the strongly interacting particles. The quarks are the most intuitively straightforward way of building up hadrons which fit into the 'SU(3)' symmetry scheme.

In 'parton pair production', by a single photon from electron-positron annihilation, it was suggested that the partons would first be produced, and would then 'clothe' themselves with other partons, spontaneously generated from the vacuum, forming hadrons of the kind which can be observed. Quarks, or any other form of parton, have never been observed, and perhaps cannot exist as free observable par- 